Rev. Téc. Ing. Univ. Zulia. Vol. 44, No. 1, Enero-Abril, 2021, 21-28

\title{
Diagnóstico del Sistema logístico de aprovisionamiento de la industria pesquera en Sancti Spíritus
}

\author{
Damaris Taydi Castillo Jiménez ${ }^{1 *}(\mathbb{D})$, Higinia Bismayda Gómez Avilés ${ }^{2}(\mathbb{D}$, Orlando de la \\ Cruz Rivadeneira $^{3} \mathbb{D}^{\mathbb{D}}$, Dariel Rivadeneira Casanueva ${ }^{1} \mathbb{D}^{\text {, Arelys López Concepción }}{ }^{1}{ }^{\mathbb{D}}$, \\ Yadira Rodríguez Fernández ${ }^{1}$
}
${ }^{1}$ Dpto. de Ingeniería Industrial, Facultad de Ciencias Técnicas y Empresariales, Universidad de Sancti Spíritus "José Martí Pérez", C.P. 60100, Cuba.
${ }^{2}$ Centro de Estudios de Energía de Procesos Industriales, Universidad de Sancti Spíritus "José Martí Pérez", C.P. 60100, Cuba.
${ }^{3}$ Empresa Pesquera de Sancti Spíritus "PESCASPIR", C.P. 60100, Cuba.
*Autor de correspondencia: damariscj@uniss.edu.cu https://doi.org/10.22209/rt.v44n1a03

Recepción: 03 de marzo de 2020 | Aceptación: 15 de septiembre de 2020 | Publicación: 01 de enero de 2021

\section{Resumen}

Las industrias pesqueras forman parte de cadenas de suministros complejas, por el limitado ciclo de vida del pescado, la alta variabilidad en la disponibilidad, calidad y cantidad de las materias primas; además, por los cambios que experimenta la calidad del producto en los diferentes procesos. El trabajo muestra los principales resultados de la aplicación de un procedimiento para el diagnóstico de la logística de aprovisionamiento a la industria pesquera, mediante un caso de estudio en una empresa de la provincia de Sancti Spíritus en Cuba. Para ello, se emplearon herramientas como el diagrama de influencias: análisis modal de fallos y efectos, análisis modal de variaciones y efecto y la matriz de escenarios. Se identificaron las perturbaciones e impacto de vulnerabilidades en la cadena de suministro, para robustecer su desempeño. Con el diseño de indicadores, se propusieron atributos para evaluar la calidad de la materia primar, a partir del método de índice de calidad.

Palabras Clave: cadena de suministro pesquera; mejora de la calidad; vulnerabilidad.

\section{Diagnosis of the logistics supply system of the fishing industry of Sancti Spiritus}

\begin{abstract}
Fishing industries are part of complex supply chains, due to the limited life cycle of fish, high variability in the availability, quality and quantity of raw materials; also for the changes experienced by the quality of the product in the different processes. The research shows the main results of the application of a procedure for the diagnosis of supply logistics to the fishing industry, through a case study in an enterprise of the Cuban province of Sancti Spíritus. For this, tools such as the influences diagram were used; failure mode and effects analysis; variant mode and effect analysis and the scenario matrix. Disturbances were identified, as well as the impact of vulnerabilities in the supply chain to strengthen its performance. With the design of indicators, attributes were proposed to evaluate the quality of the raw material, based on the quality index method.
\end{abstract}

Keywords: fishery supply chain; quality improvement; vulnerability. 


\section{Introducción}

La gestión de las cadenas de suministros facilita la integración entre la base de clientes, la red de distribución, las actividades internas de las empresas y la base de suministro [1]. Esta tiene un papel predominante en la competitividad de las empresas en términos de calidad, eficiencia, productividad y costos. En consecuencia, impera la necesidad de eliminar operaciones que no agregan valor en los procesos, minimizar los tiempos de ciclo, incrementar la productividad y minimizar los niveles de inventarios a lo largo de la cadena de suministros, y al mismo tiempo mejorar la calidad del producto, y la satisfacción al cliente tan alto como sea posible. Como resultado las cadenas de suministros son muy vulnerables a las perturbaciones debido a eventos imprevistos en cada proceso[2]. En coherencia con este planteamiento[2-4], refieren que las cadenas de suministros de los alimentos tienen características específicas que lo hacen mucho más vulnerables a las perturbaciones, por ser productos con ciclo de vida limitado, de la alta variabilidad en la disponibilidad, calidad y cantidad de las materias primas, y el hecho de que la calidad del producto puede cambiar mientras este se transforma a lo largo de los diferentes procesos. Estas vulnerabilidades hacen que la gestión de las cadenas de suministros de alimentos sea más compleja a la hora de robustecer su desempeño.

En general, la vulnerabilidad de las cadenas de suministros se refleja en el desempeño de las cadenas de suministros, la cual se cuantifica mediante indicadores claves de desempeño (ICD). El comportamiento del desempeño de las cadenas de suministros genera pérdidas poscosechas que inciden en la calidad de los productos, la productividad y los costos, entre otros[2].

En el entorno empresarial competitivo de hoy, el rendimiento de la cadena de suministro es uno de los problemas más críticos en varias industrias [5].Las cadenas de suministros son de por sí complejas, cada componente que forma parte de la misma implica detalles que son indispensables a tener en cuenta en la toma de decisiones, con la información más actualizada y precisa de todos los miembros de la misma[6].

Una cadena de suministro es una red de empresas que producen, venden y entregan un producto o servicio a un segmento de mercado predeterminado. No solo incluye a los fabricantes y proveedores, sino también a transportistas, almacenes, minoristas y los propios clientes, entre otros[7]. Según Yared Lemma y Gatew [8],las cadenas de suministros están compuestas por cuatro sistemas logísticos: aprovisionamiento, producción, comercialización y logística inversa. En las cadenas de suministros de alimentos perecederos, el sistema logístico donde se producen las pérdidas y sus causas, son diferentes para los países desarrollados y en desarrollo. En estos últimos el 64\% aproximado de las pérdidas ocurren en el sistema logístico de aprovisionamiento, debido a los límites de la tecnología y la infraestructura (como en el transporte y almacenamiento), y las técnicas para la cosecha, transporte y almacenamiento. En los países desarrollados, las pérdidas a lo largo de toda la cadena de valor de los productos alimenticios oscilan entre el 40 y el $50 \%$ del $42 \%$ del total de residuos alimenticios, y en los países en desarrollo, las pérdidas pueden ser del 30 al $50 \%$, y el $40 \%$ de las pérdidas ocurren en el nivel poscosecha y procesamiento[9].

En la etapa actual, Cuba se encuentra inmersa en un proceso de transformaciones de su economía, para sentar las bases del desarrollo económico que permita perfeccionar su sistema social socialista. Este proceso se desarrolla en el marco de una crisis económica, financiera, alimentaria, energética y ambiental a nivel internacional; en un entorno cada vez más globalizado. Al iniciarse estas transformaciones se manifiestan determinados síntomas que reflejan las incongruencias entre el concepto anterior, y el nuevo concepto de funcionamiento de la economía que se comienza a instituir. En la actualidad la gestión individual de cada empresa ya no resulta en una elevada competitividad, es necesario integrar la gestión de la cadena de suministro[10].

En la presente investigación se integran diversas herramientas para identificar los factores de ruido que inciden en el desempeño de la logística de aprovisionamiento a la industria pesquera acuícola; el tratamiento de los mismos permite robustecer el proceso y se propone el método de índice de calidad como indicador de gestión y fiabilidad del sistema.

\section{Materiales y métodos}

El procedimiento utilizadoen la Figura 1, permitió realizar un diagnóstico de la logística de aprovisionamiento a la industria pesquera de Sancti Spíritus. Donde se aplican herramientas en las diferentes etapas como análisis modal de fallos y efecto (AMFE) y análisis modal de variaciones y efecto (VMEA), que permiten identificar y clasificar las perturbaciones de la cadena, proponer los indicadores y la utilización del método de índice de calidad (QIM, por sus siglas en inglés), así como la definición de escenarios.

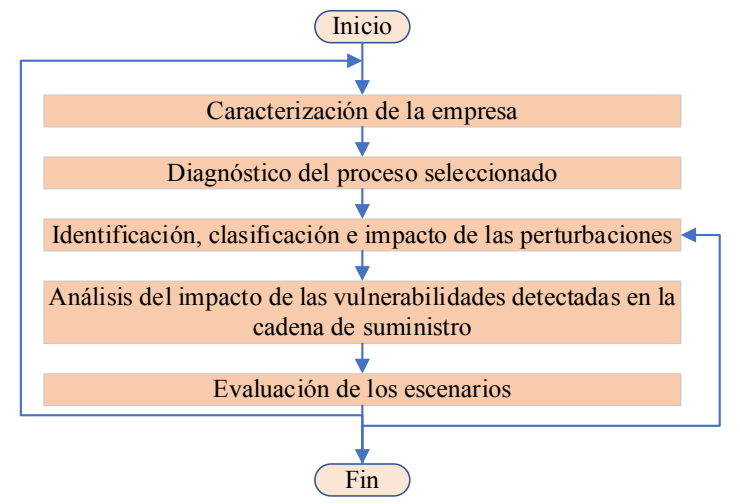

Figura 1. Procedimiento para el diagnóstico de la logística de aprovisionamiento a la industria pesquera de Sancti Spíritus [11]. 


\section{Caracterización de la empresa}

Se realizó una descripción de la empresa objeto de estudio y de la cadena de suministro.Seguidamente,se procedió a la creación del equipo de trabajoformado por especialistas con conocimientos sobre la cadena de suministro, y de los diferentes sistemas logísticos que la integran, para conducir la ejecución del procedimiento propuesto, respecto a la organización y el aporte de criterios. Los expertos brindan valoraciones y aportan recomendaciones con un máximo de competencia [12,13], lo que permitió realizar una caracterización general de la cadena de suministro y una representación gráfica, donde se identifican cada uno de los procesos, proveedores y clientes que intervienen la cadena, así como seleccionar el sistema logístico a estudiar.

\section{Diagnóstico del sistema logístico seleccionado}

Mediante una tormenta de ideas se obtuvo la información de los expertos, sobre las principales causas de los problemas que presentaba el sistema logístico. Se realizó un diagrama causa-efecto; herramienta que permite exhibir gráficamente, con detalles crecientes, todas las posibles causas relacionadas con un problema o condición, a fin de descubrir su raíz o raíces.

\section{Identificación, clasificación e impacto de las perturbaciones}

Con el análisis gráfico del proceso y la valoración de las relaciones causa y efecto de los problemas detectados en el diagnóstico, se procedió a la identificación de las perturbaciones y clasificación, para evaluar el impacto de cada perturbación en el sistema logístico seleccionado, mediante AMFE $[14,15]$.

El análisis continúo con el desarrollo del VMEA, como herramienta para determinar la magnitud de la perturbación, en términos de la variabilidad que provoca en el desempeño del proceso.

\section{Análisis del impacto de las vulnerabilidades detectadas en la cadena de suministro}

Para la identificación y relación de los procesos del sistema logístico seleccionado, se elaboró un diagrama de influencias, que permitió identificar las relaciones existentes en cada uno de los procesos del sistema logístico seleccionado. En el diagrama se representan los indicadores definidos para medir las perturbaciones. Las causas tenidas en cuentas son las identificadas en el AMFE, y el objetivo se define en el VMEA, para minimizar la variabilidad de las características de calidad.

Para el diseño de los indicadores de calidad, se propuso utilizar el QIM, que se basa en parámetros de la calidad sensorial significativos, atributos de apariencia externa para pescado crudo, con un sistema de puntuación por deméritos en una escala de 0 a $3[16,17]$.

\section{Definición de los escenarios de vulnerabilidad}

Se definieron los escenarios, y se declaran los indicadores de tiempo, cantidad y calidad mediante los indicadores de desempeño [18], en correspondencia con los resultados de los fallos identificados en el AMFE, y del análisis de variabilidad con el VMEA, para la cadena de suministro del pescado como alimento perecedero.

\section{Resultados y discusión}

La cadena de suministros de la empresa pesquera se representa en la Figura 2, donde se describen los sistemas que intervienen en el funcionamiento de la misma. Seguidamente, con la opinión de los expertos, se obtuvieron los grados de relación entre los sistemas logísticos, determinándose que el sistema a estudiar es el sistema logístico de aprovisionamiento.

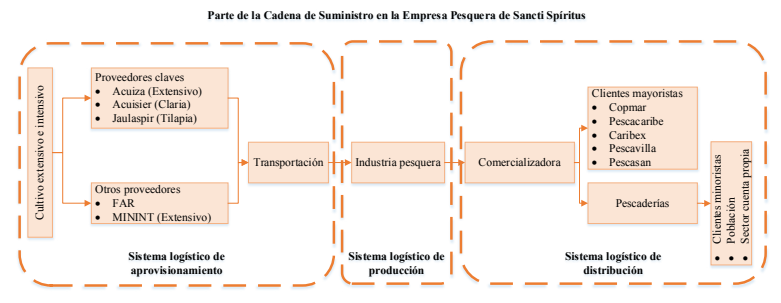

Figura 2. Representación de la cadena de suministro.

\section{Diagnóstico del sistema seleccionado}

El sistema logístico de aprovisionamiento en la empresa, se inicia en el momento de la captura y finaliza con la entrega de la materia prima a la industria. En la investigación con los expertos, se analizaron las deficiencias que inciden en el funcionamiento de este sistema, las mismas se muestran en la Figura 3.

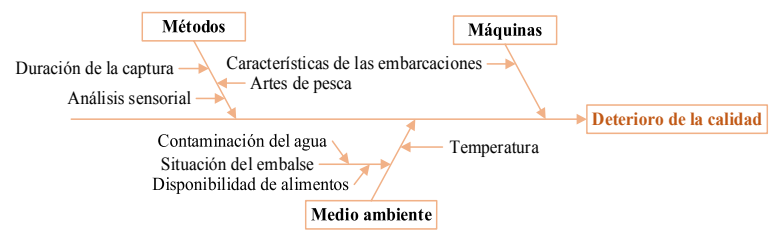

Figura 3. Deficiencias detectadas en el sistema seleccionado.

Identificación, clasificación e impacto de las perturbaciones

Para la identificación, clasificación e impacto 
de las perturbaciones, se utilizó la metodología para el AMFE, que ha permitió analizar la calidad, seguridad y/o fiabilidad del funcionamiento de cada uno de los procesos analizados. Se realizó un trabajo en grupo con los expertos, donde se identificaron los fallos potenciales, evaluando su gravedad, ocurrencia y detección, mediante las cuales, se calculó el número de prioridad de riesgo (NPR), para priorizar las causas sobre las cuales, habrá que actuar evitando que se presenten dichos modos de fallo. En la Tabla 1, se muestran los principales procesos de mayor NPR identificados.
Los resultados del VMEA muestran la contribución relativa de cada sub-KPC y NF en diagrama de Pareto (Figuras 4 y 5). Estas representaciones gráficas demuestran que el crecimiento microbiano (sub- KPC) y la temperatura (NF), son las que contribuyen más a la variabilidad de las características de calidad del pescado. En segundo lugar, se encuentra la variabilidad de la situación del embalse (sub-KPC), en correspondencia con la contaminación del agua y disponibilidad de alimentos (NF). Es por estas razones que, los esfuerzos de mejora están centrados en estas áreas. Se puede observar que

Tabla 1. Análisis modal de fallos y efectos.

\begin{tabular}{|c|c|c|c|c|c|c|}
\hline $\begin{array}{l}\text { Pasos del } \\
\text { proceso }\end{array}$ & Modo de fallo & Efectos del fallo & Causas del fallo & Control actual & NPR & Acciones correctivas \\
\hline Captura & $\begin{array}{l}\text { Deterioro de las } \\
\text { características de } \\
\text { calidad }\end{array}$ & $\begin{array}{l}\text { Contaminación } \\
\text { microbiológica de la } \\
\text { materia prima }\end{array}$ & $\begin{array}{c}\text { Altas } \\
\text { temperaturas }\end{array}$ & Análisis visual & 504 & $\begin{array}{c}\text { Cumplimiento de las normas } \\
\text { de nevado basado en la } \\
\text { temperatura ambiente }\end{array}$ \\
\hline $\begin{array}{l}\text { Recepción en el } \\
\text { punto de pesca }\end{array}$ & $\begin{array}{l}\text { Deterioro de las } \\
\text { características de } \\
\text { calidad }\end{array}$ & $\begin{array}{l}\text { Contaminación } \\
\text { microbiológica de la } \\
\text { materia prima }\end{array}$ & $\begin{array}{c}\text { Altas } \\
\text { temperaturas }\end{array}$ & $\begin{array}{c}\text { Evaluación } \\
\text { sensorial } \\
\text { (método de } \\
\text { índice calidad) }\end{array}$ & 846 & $\begin{array}{c}\text { Cumplimiento de las normas } \\
\text { de nevado basado en la } \\
\text { temperatura ambiente }\end{array}$ \\
\hline $\begin{array}{l}\text { Traslado hacia } \\
\text { a la industria }\end{array}$ & $\begin{array}{l}\text { Problemas de } \\
\text { transportación de } \\
\text { la materia prima }\end{array}$ & $\begin{array}{l}\text { Contaminación } \\
\text { microbiológica de la } \\
\text { materia prima }\end{array}$ & $\begin{array}{l}\text { Contaminación } \\
\text { del medio } \\
\text { Altas } \\
\text { temperaturas }\end{array}$ & $\begin{array}{c}\text { Evaluación } \\
\text { sensorial } \\
\text { (método de } \\
\text { índice calidad) }\end{array}$ & 846 & $\begin{array}{l}\text { Chequear que en el traslado } \\
\text { de la materia prima se } \\
\text { cumpla con las normas } \\
\text { establecidas de nevado. } \\
\text { Utilizar carros isotérmicos, } \\
\text { cajas plásticas y nevadas } \\
\text { correctamente }\end{array}$ \\
\hline
\end{tabular}

NPR: índice de prioridad de riesgo.

Tabla 2. Resultados de la aplicación del análisis modal de variaciones y efecto.

\begin{tabular}{cccccccc}
\hline $\begin{array}{c}\text { Características } \\
\text { claves del } \\
\text { producto KPC }\end{array}$ & Sub-KPC & Sensibilidad & $\begin{array}{c}\text { Factor de } \\
\text { ruido (NF) }\end{array}$ & Sensibilidad & $\begin{array}{c}\text { Tamaño } \\
\text { variación NF }\end{array}$ & $\begin{array}{c}\text { Prioridad } \\
\text { del riesgo de } \\
\text { la variación } \\
\text { VRPN (NF) }\end{array}$ & $\begin{array}{c}\text { Prioridad } \\
\text { del riesgo de } \\
\text { la variación } \\
\text { (Sub-KPC) }\end{array}$ \\
\hline $\begin{array}{c}\text { Rendimiento } \\
\text { Industrial }\end{array}$ & $\begin{array}{c}\text { Procesamiento } \\
\text { industrial }\end{array}$ & 10 & $\begin{array}{c}\text { Estado de la } \\
\text { tecnología } \\
\text { Capacidad } \\
\text { industrial }\end{array}$ & 9 & 9 & 7 & 230 \\
\hline
\end{tabular}

NF: factor de ruido, VNPR: variación de la prioridad del riesgo

Para definir los factores que inciden en la variabilidad del proceso se utilizó el VMEA, aplicando una tormenta de ideas para identificar las características claves del producto (KPC) y los Sub-KPC, por sus siglas en inglés. Se selecciona como KPC la calidad del pescado, dada las necesidades de la industria. La descripción de cada Sub KPC se muestra en la Tabla 2, y permite comprender las características que más contribuyen a las variaciones del KPC. existen otras sub-KPC y NF que su contribuciónrelativa sobre las KPC no fuesignificativa, pero tienen incidencia directa y desfavorable en los índices de consumo de los productos de frutas y vegetales; por ende, en la eficiencia del proceso, se procedió a realizar el VMEA, tomando como KPC la calidad del pescado. De esta forma, se visualiza la situación que presenta el embalse por la contaminación del agua existente, y la disponibilidad de alimentos también 
genera variabilidad. Por lo que, se hace necesario trabajar en ellas, para mejorar el aseguramiento de la calidad de la logística de aprovisionamiento de la empresa.

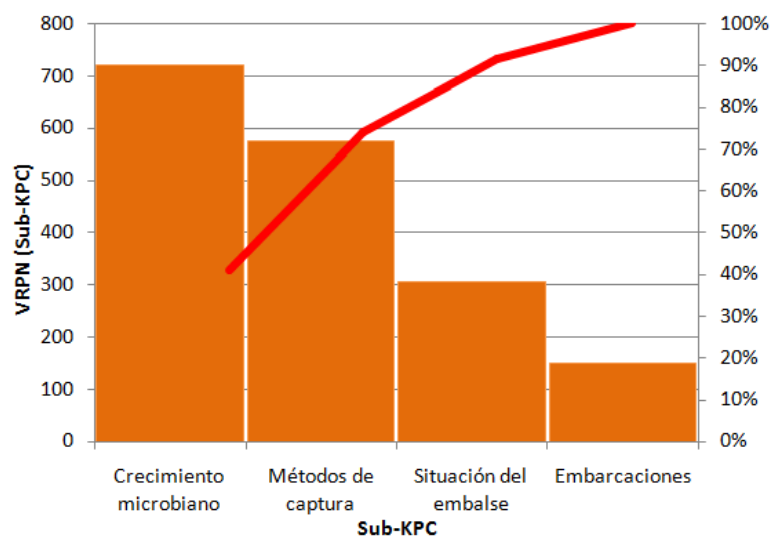

Figura 4. Contribución relativa de la sub- KPC para la calidad del pescado.

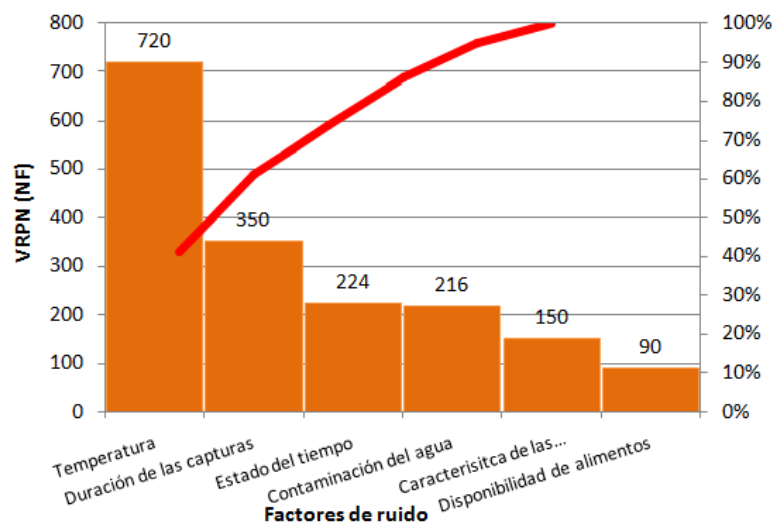

Figura 5. Contribución relativa de los NF para la calidad del pescado.

Análisis del impacto de las vulnerabilidades detectadas en la cadena de suministro

Para el análisis del impacto de las vulnerabilidades se hace una representación a través del diagrama de influencias, como se muestra en la Figura 6. Se observa la relación que existe entre: las causas de las perturbaciones, la relación de cada una de ellas con los indicadores definidos, y la relación directa del indicador de calidad. De esta forma, se visualiza el tipo de relación de las variables con el sistema, y se establecen las acciones de mejora que se corresponden con la cuantificación de estas relaciones, para minimizar la variabilidad en las características de calidad.

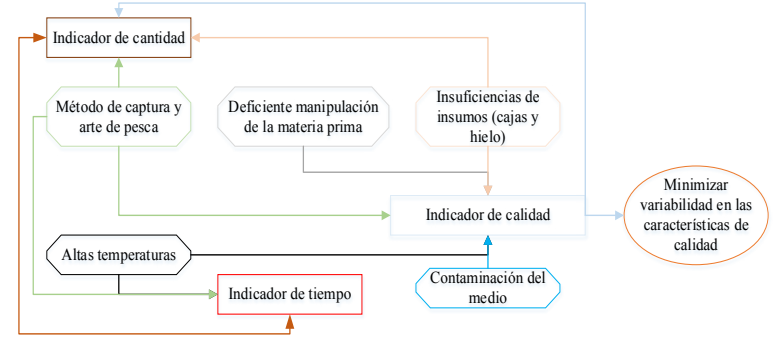

Figura 6. Relaciones de los NF y Sub-KPC con el sistema que minimizan la variabilidad en las características de calidad.

Para el análisis del impacto de las vulnerabilidades de la cadena de suministro detectadas en el VMEA, se realiza una propuesta de indicadores de tiempo, magnitud y calidad, que se muestran en la Tabla 3. La propuesta de indicadores de tiempo, magnitud, para el análisis del impacto de las vulnerabilidades de la cadena de suministro detectadas en el VMEA, se detalla en la Tabla 3. Los atributos a considerar como indicadores de calidad del producto para utilizar el QIM, se incluyen en la Tabla 4.

Los atributos definidos fueron considerados como los parámetros más importantes para realizar el análisis sensorial, a través del QIM, para determinar la frescura del pescado, con puntuaciones que van de 0 a 3 (olor) y de 0 a 2 (otros atributos), en dependencia de la puntuación de las características de cada una de ellas.La suma total oscila entre 0 (frescura total) y 16 (pérdida total de frescura).

\section{Evaluación de los escenarios}

La evaluación del comportamiento de los escenarios se realiza de forma cualitativa a través de los fallos identificados en el AMFE, y la variabilidad que brinda el AMFE, se representa en la Tabla 5, la cualpermite mostrar cómo inciden estas perturbaciones y en qué régimen de trabajo se clasifican. Se toman como referencia para ubicar los escenarios en la matriz, según los fallos y los $\mathrm{NF}$ determinados los meses actuales, ya que la tendencia es obtener altos volúmenes de pescado y con altas temperaturas.

En el proceso de captura se identifican fallos en los métodos implementados, estos traen consigo daños mecánicos si se prolonga la duración de la captura y las embarcaciones no tienen las condiciones necesarias para el nevado del pescado, y al estar expuestos a altas temperaturas, aumenta la contaminación microbiana. Estas perturbaciones se sitúan en el escenario E6 en un régimen alterado, con respuesta preventiva y reducida. 
Tabla 3. Indicadores de tiempo, cantidad y calidad.

\begin{tabular}{|c|c|c|c|}
\hline Proceso & Expresión de cálculo & Evaluación & Leyenda \\
\hline $\begin{array}{l}\text { Recepción en el } \\
\text { punto de pesca }\end{array}$ & $\mathrm{IC}=\frac{\mathrm{CLNMIC}}{\mathrm{TL}}$ & $\begin{array}{l}\text { Bien: } \mathrm{IC}=0 \\
\text { Mal: } \mathrm{IC}>0\end{array}$ & $\begin{array}{c}\text { Indicador de cantidad (IC) } \\
\text { Cantidad de lotes que no cumplen con el índice } \\
\text { de calidad (CLNMIC) } \\
\text { Total de lotes (TL) }\end{array}$ \\
\hline Punto de acopio & $\mathrm{IT}=\frac{\mathrm{CLNTDCD}}{\mathrm{TL}}$ & $\begin{array}{l}\text { Bien: } \mathrm{IT}=0 \\
\text { Mal: } \mathrm{IT}>0\end{array}$ & $\begin{array}{c}\text { Indicador de tiempo (IT) } \\
\text { Cantidad de lotes que incumplen con el tiempo } \\
\text { definido en la curva de deterioro (CLNTDCD = } \\
\text { Total de lotes (TL) }\end{array}$ \\
\hline Punto de acopio & $\mathrm{IQ}=\frac{\mathrm{CPCIQ}}{\mathrm{CTC}}$ & $\begin{array}{l}\text { Bien: } I Q=0 \\
\text { Mal: } I Q>0\end{array}$ & $\begin{array}{c}\text { Indicador de Calidad (IQ) } \\
\text { Cantidad de pescado que no cumplen el índice de } \\
\text { calidad (CPCIQ) } \\
\text { Cantidad total capturada (CTC) }\end{array}$ \\
\hline $\begin{array}{l}\text { Recepción en la } \\
\text { industria }\end{array}$ & $\mathrm{IQ}=\frac{\mathrm{CPCIQ}}{\mathrm{CTR}}$ & $\begin{array}{l}\text { Bien: } I Q=0 \\
\text { Mal: } I Q>0\end{array}$ & $\begin{array}{c}\text { Indicador de Calidad (IQ) } \\
\text { Cantidad de pescado que no cumplen el índice de } \\
\text { calidad (CPD) } \\
\text { Cantidad total recibida (CTR) }\end{array}$ \\
\hline $\begin{array}{l}\text { Recepción en la } \\
\text { industria }\end{array}$ & $\mathrm{IT}=\frac{\text { CLNTDCD }}{\mathrm{TL}}$ & $\begin{array}{l}\text { Bien: } I Q=0 \\
\text { Mal: } I Q>0\end{array}$ & $\begin{array}{c}\text { Indicador de tiempo (IT) } \\
\text { Cantidad de lotes que incumplen con el tiempo } \\
\text { definido en la curva de deterioro (CLNTDCD = } \\
\text { Total de lotes (TL) }\end{array}$ \\
\hline
\end{tabular}

Tabla 4. Atributos de calidad para medir indicador de calidad.

\begin{tabular}{ccc}
\hline \multicolumn{2}{c}{ Parámetros de calidad } & Puntuaciones \\
\hline \multirow{3}{*}{ Apariencia general } & Color & $0-2$ \\
& Olor & $0-3$ \\
& Piel & $0-2$ \\
& Escamas & $0-2$ \\
Ojos & Forma & $0-1$ \\
& Pupila & $0-2$ \\
Branquias o agallas & Cornea & $0-2$ \\
Índice de Calidad & Color & $0-2$ \\
\hline
\end{tabular}

El proceso de recepción en el punto de pesca se ubica en el escenario E5, bajo un régimen alterado, ya que se toma el tiempo medio y los niveles de cantidad son altos, por lo que deben analizarse las artes de pesca utilizadas. Para garantizar la calidad de la materia prima en ese punto del proceso, deben asegurarse los insumos necesarios, como cajas plásticas y el hielo para el nevado. El traslado hacia la industria se ubica en los escenarios E1 y E3, bajo un régimen normal, con respuesta reducida, tiempo y cantidad media. Para garantizar la calidad de la materia prima,debe asegurarse una adecuada manipulación, así como los insumos de hielo y cajas plásticas, para mantener la temperatura adecuada. Los escenarios pueden variar bajo una situación dada en un momento determinado, como son las condiciones climatológicas, altos volúmenes de captura, que no se cuente con los insumos necesarios, etre otros.
Tabla 5. Matriz de relación de los indicadores de tiempo y cantidad.

\begin{tabular}{|c|c|c|c|}
\hline 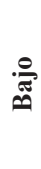 & $\begin{array}{c}\text { E0 } \\
\text { Régimen } \\
\text { ideal } \\
\text { La zona de } \\
\text { robustez }\end{array}$ & $\begin{array}{c}\text { E1 } \\
\text { Régimen } \\
\text { normal } \\
\text { Respuesta } \\
\text { reducida }\end{array}$ & $\begin{array}{c}\text { E4 } \\
\text { Interrupción de } \\
\text { régimen } \\
\text { Respuesta } \\
\text { reducida }\end{array}$ \\
\hline 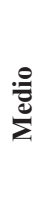 & $\begin{array}{c}\text { E3 } \\
\text { Régimen } \\
\text { normal } \\
\text { Respuesta } \\
\text { preventiva }\end{array}$ & $\begin{array}{c}\text { E1, E3 } \\
\text { Traslado hacia } \\
\text { la industria } \\
\text { Régimen normal } \\
\text { Respuesta } \\
\text { reducida (T,I,M) }\end{array}$ & $\begin{array}{c}\text { E5 } \\
\text { Recepción del } \\
\text { punto de pesca } \\
\text { Régimen alterado } \\
\text { Respuesta reducida } \\
\text { (MCU,T,I,M) }\end{array}$ \\
\hline$\stackrel{\rho}{\frac{\rho}{4}}$ & $\begin{array}{c}\text { E3 } \\
\text { Régimen } \\
\text { alterado } \\
\text { Respuesta } \\
\text { preventiva }\end{array}$ & $\begin{array}{c}\text { E2, E6 } \\
\text { Régimen } \\
\text { alterado } \\
\text { Respuesta } \\
\text { preventiva }\end{array}$ & $\begin{array}{c}\text { E6 } \\
\text { Proceso de captura } \\
\text { Régimen alterado } \\
\text { Respuesta } \\
\text { preventiva } \\
\text { y reducida } \\
\text { (MCU,T,I,M) }\end{array}$ \\
\hline & Bajo & Medio & Alto \\
\hline \multicolumn{4}{|c|}{ Indicadores de calidad } \\
\hline
\end{tabular}

\section{Conclusiones}

Los gestores de las cadenas de suministros, como se hace énfasis en la presente investigación, deben estar mejor equipados con los métodos de medición y gestión de la vulnerabilidad de estascadenas El procedimiento diseñado permite integrar herramientas 
científicas con el objetivo de contribuir a evaluar los niveles de vulnerabilidad de la cadena de suministros y gestionar de manera proactiva la respuesta a considerar para cada tipo de escenario. El procedimiento estudia las vulnerabilidades de la cadena de suministros, dependiendo de la cantidad y el tiempo de exposición a altas temperaturas de las capturas, y su impacto en las características de calidad, evaluadas mediante el análisis sensorial, logrando de esta manera, una relación directa en la gestión de los diferentes procesos. De ese modo, la alta dirección obtiene información más confiable sobre la "salud" de la empresa, y puede evaluar si la cadena de suministros se gestiona de manera eficaz y eficiente, con una filosofía de mejora continua de la calidad.

\section{Agradecimientos}

Los autores agradecen a la dirección de la empresa pesquera de Sancti Spíritus, la posibilidad de realizar la investigación y contribuir a mejorar las vulnerabilidades detectadas en el aprovisionamiento de la cadena de suministro.

\section{Referencias bibliográficas}

[1] Bastas A. y Liyanage K.:"Sustainable supply chain quality management: A systematic review". Journal of Cleaner Production, Vol. 181, (2018) 726-744.

[2] Vlajic J. V., Van der Vorst J., y Djurdjevic D." Influence of product and business environment characteristics on managing supply chain vulnerability-a conceptual foundation". International Journal for Traffic \& Transport Engineering, Vol. 9, No. 3 (2019).

[3] Banasik A., Bloemhof-Ruwaard J. M., Kanellopoulos A., Claassen G., y Van der Vorst J. G.: "Multi-criteria decision making approaches for green supply chains: A review". Flexible Services and Manufacturing Journal, Vol. 30, No. 3 (2018) 366-396.

[4] Banasik A., Kanellopoulos A., Claassen G., BloemhofRuwaard J. M., y Van der Vorst J. G.: "Assessing alternative production options for eco-efficient food supply chains using multi-objective optimization". Annals of Operations Research, Vol. 250, No. 2 (2017) 341-362.

[5] Balfaqih H., Nopiah Z. M., Saibani N., y Al-Nory M. T.: "Review of supply chain performance measurement systems": 1998-2015. Computers in Industry, Vol. 82, (2016) 135-150.

[6] Ogunwolu L., Ibidapo-Obe O., y Onyedikam C.: "Modelling and analysis of a supply chain with supply, production and distribution reliability considerations". (2014) 41-51.
[7] Icarte Ahumada G. A.: "Aplicaciones de inteligencia artificial en procesos de cadenas de suministros: una revisión sistemática". Revista chilena de ingeniería, Vol. 24, No. 4 (2016) 663-679.

[8] Yared Lemma D. K. y Gatew G.: "Loss in Perishable Food Supply Chain: An Optimization Approach Literature Review". International Journal of Scientific \& Engineering Research, Vol. 5, No. 5 (2014).

[9] Shukla M. y Jharkharia S.: "Agri-fresh produce supply chain management: a state-of-the-art literature review". International Journal of Operations \& Production Management, Vol. 33, No. 2 (2013) 114158.

[10] Gómez-Acosta M. I., Acevedo-Suárez J. A., Pardillo-Baez Y., López-Joy T., y Lopes-Martínez I.:"Caracterización de la Logística y las Redes de Valor en empresas cubanas en Perfeccionamiento Empresarial". Ingeniería Industrial, Vol. 34, No. 2 (2013) 212-226.

[11] Vlajic J. V., Van der Vorst J. G. y Haijema R.: "A framework for designing robust food supply chains". International Journal of Production Economics, Vol. 137, No. 1 (2012) 176-189.

[12] Soorianathasundaram, K., Narayana, C.K. and Paliyath, G.: Bananas and Plantains. Encyclopedia of Food and Health.Oxford: Academic Press, (2016) 320-327.

[13] Hernández Nariño A.:"Contribución a la gestión y mejora de procesos en instalaciones hospitalarias del territorio matancero (Grado científico de Doctor en Ciencias Técnicas)". Universidad de Matanzas. Matanzas, Cuba, (2010).

[14] Medina León A.: "Selección de los procesos claves de una instalación hotelera como parte de la gestión y mejora de procesos". Revista Cubana de Investigaciones Turísticas, Vol. 7, No. 3 (2008).

[15] Gutierrez P. H. y de la Vara Salazar R.: "Control estadístico de la calidad y Seis Sigma” (2009).

[16] Santos-Rubio M., Marín-Gil R., Muñoz-de la Corte R., Velázquez-López M., Gil-Navarro M., y BautistaPaloma F.: "Análisis modal de fallos y efectos aplicado a la elaboración de citostáticos intravenosos". Revista de Calidad Asistencial, Vol. 31, No. 2 (2016) 106-112.

[17] Billar dos Santos A., Mitsui M., Macedo E., y LapaGuimaraes J.: "Development of Quality Index Method (QIM) scheme for Acoupa weakfish (Cynoscion acoupa)". LWT-Food Sci Technol, Vol. 57, No. 1 (2014) 267-275. 
[18] Hyldig G. y M. Green-Petersen D.: “Quality Index Method-an objective tool for determination of sensory quality". Journal of aquatic food product technology, Vol. 13, No. 4 (2005) 71-80.
[19] Zúñiga B. E., Ordóñez M. C., Ayala J. E., Santillán D. y Recalde C. G.: "Quality of service 2.5G for hydrometeorological network in the Chimborazo province, Ecuador". Rev. Téc. Ing. Univ. Zulia. Vol. 39, No. 3, (2016) 130-136. 


\section{REVISTA TECNICA}

DE LA FACULTAD DE INGENIERIA

UNIVERSIDAD DEL ZULIA

Vol. 44. N`1, Enero - Abril 2021, pp. 04 - 58

Esta revista fue editada en formato digital y publicada en Diciembre de 2020, por el Fondo Editorial Serbiluz, Universidad del Zulia. Maracaibo-Venezuela

www.luz.edu.ve

www.serbi.luz.edu.ve

www.produccioncientificaluz.org 